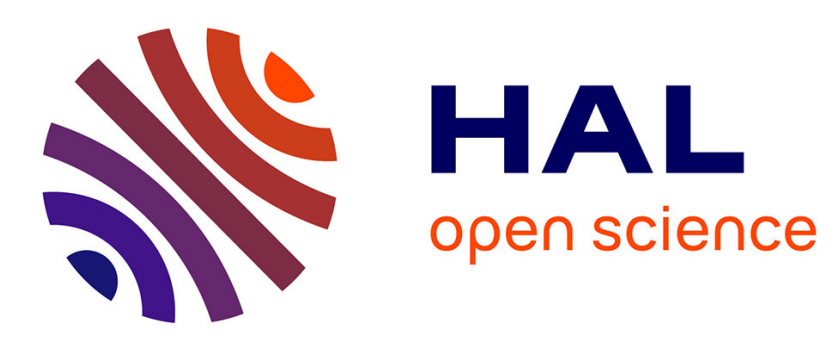

\title{
Naissance en temps de Covid 19
}

\author{
Marie-Laure Deneffe Dobrzynski
}

\section{To cite this version:}

Marie-Laure Deneffe Dobrzynski. Naissance en temps de Covid 19 . Recherches \& éducations, 2020, Quelle éducation avec la Covid-19, HS, 10.4000/rechercheseducations.11353 . hal-03512201

\section{HAL Id: hal-03512201 \\ https://hal.science/hal-03512201}

Submitted on 5 Jan 2022

HAL is a multi-disciplinary open access archive for the deposit and dissemination of scientific research documents, whether they are published or not. The documents may come from teaching and research institutions in France or abroad, or from public or private research centers.
L'archive ouverte pluridisciplinaire HAL, est destinée au dépôt et à la diffusion de documents scientifiques de niveau recherche, publiés ou non, émanant des établissements d'enseignement et de recherche français ou étrangers, des laboratoires publics ou privés. 


\title{
OpenEdition
}

Journals

\section{Recherches \& éducations}

HS • Juillet 2020 :

Quelle éducation avec la Covid-19

Une éducation au 3ème corps

\section{Naissance en temps de Covid 19}

Birth in time of Covid 19

\author{
MARIE-LAURe DenefFe DobRZYŃSKI \\ https://doi.org/10.4000/rechercheseducations.11353
}

\section{Résumés}

Français English

Cet article propose une analyse des pratiques discursives autour de la naissance pendant le confinement lié à la pandémie de Covid 19. Cette analyse fait émerger un recul majeur du droit des femmes à être accompagnées de la personne de leur choix lors de leurs suivis de grossesse, de l'accouchement et de l'hospitalisation en maternité. Ce travail débouche sur des questions concernant les modèles de santé génésique qui ont sous-tendu non seulement les discours sur les pratiques mais également la pratique issue des discours.

This article is an analysis of the discursive practices surrounding the moment of birth during confinement because of the Covid-19 pandemic. This analysis reveals a major decline in the rights of women to be accompanied by the person of their choice during their pregnancy, during childbirth, and maternity hospital follow-ups. This work leads to major questions concerning the models of reproductive health which have underpinned not only the discourse on practices but also the practice resulting from discourse.

\section{Entrées d'index}

Mots-clés : confinement lié à la pandémie de covid 19, droits sexuels et reproductifs, droits des femmes, droit à l'accompagnement autour de la naissance, violences obstétricales, ethique médicale, modèles en santé génésique

Keywords : containment linked to the covid 19 pandemic, sexual and reproductive rights, women's rights, right to support around birth, obstetrical violence, medical ethics, reproductive health models

\section{Introduction}


1 Le droit à être accompagnée de la personne de son choix lors du suivi de grossesse, de l'accouchement et des suites de la naissance est reconnu par l'Organisation Mondiale de la Santé (OMS) comme l'un des piliers fondamentaux pour le droit de toute femme à une expérience positive et sans risques de l'accouchement (WHO, 2016, 2018). Le 18 mars 2020, l'OMS a rappelé que le droit à être accompagnée par la personne de son choix devait être respecté pour «toutes les femmes enceintes, y compris celles dont l'infection par le virus de la COVID-19 est confirmée ou soupçonnée » (WHO 2020).

En France, le dernier Plan Périnatalité 2005-2007 place la sécurité émotionnelle des femmes enceintes en objectif primordial (Molénat, F., 2007) et précise que les futurs parents doivent pouvoir être entendus par les professionnels de la naissance pour les modalités de suivi de la grossesse et le contexte de l'accouchement et, au-delà, que soit privilégiée leur participation à l'élaboration de la politique périnatale. Il précise également que les futurs parents doivent pouvoir bénéficier de séances de préparation à la naissance et à la parentalité délivrées par des professionnels de la naissance en milieu hospitalier ou en médecine de ville. (Puech, F., 2007)

Or lors du confinement lié à la pandémie de Covid 19, c'est-à-dire durant la période du 17 mars au 11 mai 2020, un ensemble de recommandations à valeur restrictive a été posé autour de la naissance. Se pose alors la question de la genèse et de l'application de ces recommandations et de leur effet sur les droits des femmes cités précédemment et, plus globalement, des futurs ou jeunes parents et des nouveau-nés.

L'objectif de cet article est de réaliser une analyse des pratiques discursives émergentes autour de la naissance pendant le confinement. A partir d'une analyse de contenu (Bardin, L., 2013) de différents écrits, il s'agit dans cet article de comprendre la naissance pendant la pandémie de Covid 19, comme un dispositif ${ }^{1}$ au sens foucaldien (Foucault, M., 1994 : 299, 300) en s'appuyant sur une analyse des pratiques discursives relatives au droit des femmes à l'accompagnement pendant le confinement. Les publications analysées sont de différents types : communiqués d'organisations professionnelles et d'associations d'usagers, contenus de sites internet, articles journalistiques, rapports d'enquêtes.

\begin{tabular}{|c|c|c|}
\hline Ecrits analysés & Date de publication & Nombre \\
\hline Communiqués d'organisations professionnelles & \multirow{5}{*}{$\begin{array}{l}\text { Entre le } 17 \text { mars et le } 11 \text { mai } \\
2020\end{array}$} & 15 \\
\hline Communiqués d'associations d'usagers & & 7 \\
\hline Articles journalistiques & & 20 \\
\hline Contenus de sites internet de maternités & & 19 \\
\hline $\begin{array}{l}\text { Contenus de sites internet et de forums dédiés aux } \\
\text { parents }\end{array}$ & & 30 \\
\hline Rapports d'enquêtes & Juin et juillet 2020 & 2 \\
\hline
\end{tabular}

Tableau 1 : Typologie des écrits

2 A noter que dans cet article, il est fait référence au droit spécifique des femmes à l'accompagnement pendant la naissance, sachant que de nombreux articles ont déjà mis en évidence le recul majeur d'autres droits sexuels et reproductifs des femmes pendant le confinement, notamment en ce qui concerne les inégalités et violences liées au genre et l'accès à l'Interruption Volontaire de Grossesse.

\section{Recommandations du CNGOF}

Les 27 et 30 mars puis le 27 avril 2020, le CNGOF (Collège National des Gynécologues et Obstétriciens Français) publiait des recommandations pour le suivi de la grossesse et de l'accouchement durant la crise sanitaire. Nous présentons les principaux éléments indiquant qu'est préconisée, jusqu'au 27 avril, l'éviction de l'accompagnant.e durant les suivis de grossesse, la première partie du travail et lors du 
séjour en maternité.

Le communiqué émis le 27 mars affirme l'importance de « garder à l'accouchement sa composante familiale et humaine " puis " recommande d'accepter le père en salle de naissance à partir de la phase active du travail » en précisant qu' " en salle d'accouchement », il ne pouvait y avoir " de sortie du père de la salle pendant toute la durée de l'accouchement sous aucun prétexte (boire, manger, fumer) » et qu' " en cas de sortie, il doit quitter définitivement l'hôpital ", que " le père doit sonner pour aller aux toilettes » et qu'il doit quitter définitivement l'hôpital deux heures après la naissance et ne peut revenir que pour « chercher sa conjointe » le jour de la sortie de maternité de la mère et du nouveau-né. (CNGOF, 27 mars 2020)

Le communiqué émis le 30 mars $^{2}$ préconise l'interdiction de la présence de tout accompagnant.e lors des consultations et des échographies ${ }^{3}$ (« il ne peut y avoir d'accompagnant lors de ces consultations ou échographies ») ainsi que lors du séjour en maternité de la mère et du nouveau-né (« aucune visite n'est autorisée et le conjoint ne peut accompagner la mère et l'enfant dans la chambre de suites de couches ») et restreint la présence de l'accompagnant.e durant l'accouchement (« la présence de l'accompagnant est parfois acceptée durant l'accouchement, sous réserve de conditions drastiques à respecter »). Il est également dit que « le suivi de grossesse doit se faire en priorité par téléconsultation. Si une consultation est indispensable (échographies de grossesse et pelviennes pour les pathologies gynécologiques), la patiente doit consulter les gynécologues ou les sages-femmes libéraux » et ne venir à l'hôpital que si nécessaire. Le CNGOF couplait ces recommandations avec une " mise en garde contre l'accouchement au domicile et les risques associés ». (CNGOF, SYNGOF, 30 mars 2020)

Le 27 avril, le CNGOF publiait un nouveau communiqué, assouplissant certaines de ces recommandations, « dans le cadre de la préparation du déconfinement ». Sont décrits en préambule « la particularité qu'est l'événement de vie que représente une naissance, l'importance pour un couple de préparer ensemble les différents temps qui la précèdent et qui la suivent et le nécessaire accompagnement autour des enjeux psychiques de la périnatalité ». Puis est ré-envisagée la " présence possible de l'accompagnant aux consultations indispensables, lors des échographies, à l'accouchement ou lors du séjour en post-partum en fonction des conditions locales 4 » 5 . Les mesures de « confinement » de l'accompagnant.e en salles de naissances restent les mêmes que celles préconisées le 27 mars et s'étendent à la période des suites de naissance. (CNGOF, 27 avril 2020)

Le contenu de ces recommandations montre qu'est préconisée jusqu'au 27 avril l'éviction de l'accompagnant.e lors des consultations prénatales, des échographies, pendant la première phase de travail et durant l'hospitalisation des mères et des nouveau-nés dans les services de suites de naissance. La possibilité pour les femmes d'être accompagnées par la personne de leur choix est par ailleurs soumise à conditions pendant la phase active du travail, l'accouchement et les deux heures qui suivent la naissance.

Le communiqué du 27 mars évoque "le père ", choix de mot restrictif ne prenant pas en compte les couples homosexuels, les femmes menant une grossesse sans conjoint.e. ou pouvant choisir d'être accompagnées par une personne autre que son/sa conjoint.e. Le communiqué du 30 mars évoque « le conjoint » qui pourrait refléter à nouveau une vision normative du couple et de la famille tout en laissant la porte ouverte à envisager que « le conjoint » puisse englober les éventuelles conjointes (normes grammaticales genrées). Le communiqué du 27 avril évoque enfin « l'accompagnant », terme pouvant englober l'ensemble des cas de figure se présentant ${ }^{6}$.

On peut enfin relever une tension entre des préambules affirmant l'importance de la sécurisation émotionnelle des futurs parents, en concordance avec les préconisations du Plan Périnatalité 2005-2007, et le haut degré des restrictions posées qui limitent de facto l'accès des futurs parents à cette sécurité émotionnelle (Bohren, M.A. et al., 2017). Le droit des femmes à être accompagnées de la personne de leur choix, droit qui a été rappelé par l'OMS dès le lendemain du confinement, n'est pas évoqué. 


\section{Etat des lieux des pratiques pendant le confinement}

Les résultats de l'analyse de contenus de communiqués d'organisations professionnelles et d'associations d'usagers, d'articles journalistiques, de sites internet des maternités, de sites internet et de forums dédiés aux parents ainsi que les résultats de l' « enquête Covid-SF » (Rousseau, A. et al., Juin 2020)7 et les résultats de l' « enquête sur la grossesse, l'accouchement et le post-partum pendant l'épidémie de Covid-19 » (Tou.te.s Contre les Violences Obstétricales et Gynécologiques., Juillet 2020 $)^{8}$ montrent que :

- les séances collectives de préparation à la naissance et à la parentalité ont fréquemment été interrompues aussi bien en milieu hospitalier qu'en médecine de ville ${ }^{9}$,

- la téléconsultation a été privilégiée pour les consultations prénatales et la préparation à la naissance et à la parentalitée,

- la plupart des femmes ont été reçues seules, sans autorisation de présence de l'accompagnant.e, lors du suivi de grossesse (échographie, consultations prénatales...) et en préparation individuelle à la naissance et à la parentalité (si maintenue à titre exceptionnel en présentiel) ${ }^{11}$,

- les accompagnant.e.s ont pu ne pas être accepté.e.s lorsque la femme était hospitalisée en cours de grossess $\mathrm{e}^{12}$, lors de la première phase de travail voire lors de l'accouchement ${ }^{13}$ ainsi que lors des suites de naissance ${ }^{14}$ et devoir accepter des mesures de « confinement » lorsqu'ils.elles étaient accepté.e.s.

Les annulations de séances collectives ont généré un accès insuffisant à la préparation à la naissance et à la parentalité : l'ensemble des futurs parents prévoyant de suivre des séances collectives n'a pu être accueilli en séances individuelles, d'autant plus que des séances individuelles de préparation à la naissance ont également pu être annulées ou reportées ${ }^{15}$. La pratique de téléconsultations en préparation à la naissance et l'éviction des accompagnant.e.s lors de rendez-vous en présentiel ont également pu empêcher la poursuite de certaines formes de préparation à la naissance et à la parentalitée ${ }^{16}$. Les sages-femmes " ont considéré que les patientes étaient, pendant le confinement, moins bien informées ou préparées » (Rousseau, A. et al., 2020) ${ }^{17}$.

Ces modifications des pratiques autour de la naissance vont à l'encontre du droit de toutes les femmes, susceptibles ou non d'être infectées par le virus de la Covid 19, à être accompagnées de la personne de leur choix lors du suivi de grossesse, de l'accouchement et des suites de la naissance (WHO, 2020). Elles vont également à l'encontre des préconisations du Plan Périnatalité 2005-2007 pour l'accès des futurs parents à des séances de préparation à la naissance et à la parentalité et plus globalement pour leur accès à des conditions favorisant leur sécurisation émotionnelle et leur autonomie.

\section{Influence des recommandations}

Le CNGOF a été la principale organisation professionnelle à émettre des recommandations préconisant l'éviction des accompagnant.e.s autour de la naissance ${ }^{18}$. L'analyse des pratiques discursives semble montrer que ces recommandations ont servi, après leur publication, de référence lors de l'application de mesures restrictives quant à la présence des accompagnant.e.s dans les maternités et en médecine de ville. Un article informant les médecins généralistes du contenu de ces recommandations titrera ainsi «Suivi de grossesse et accouchement : les gynécos fixent les règles en période épidémique » (Evrard N., 1er avril 2020). Les recommandations du CNGOF ont par ailleurs pu être reprises mot pour mot sur différents sites internet de centres hospitaliers annonçant aux usagers les mesures d'éviction des accompagnant.e.s. (CHU Rouen. Maternité Charles Nicolle, 31 mars 2020). Pour la période précédant la publication des communiqués du CNGOF, l'analyse semble montrer que ce sont les 
échanges informels entre pairs gynécologues obstétrcien.ne.s qui ont eu une influence prépondérante dans l'établissement des protocoles en maternités, avec notamment des échanges d'expériences entre des gynécologues obstétricien.ne.s chef.e.s de service et leurs collègues des maternités du Grand Est. Le communiqué publié le 30 mars par le CNGOF semble corroborer cela, indiquant que « dans chaque région, les maternités élaborent actuellement leur protocole de prise en charge des accouchements. Ils sont semblables au protocole de référence qui a été élaboré en Alsace, région la plus durement touchée par l'épidémie de COVID-19 à ce jour » (CNGOF, 30 mars 2020).

L'analyse des sites internet des maternités, des sites internet et forums dédiés aux parents ainsi que des articles de presse relayant des témoignages de vécus d'accouchements pendant le confinement semble montrer que les principaux écarts ayant pu être observés entre les recommandations du CNGOF et les mesures mises en œuvre dans les maternités ont consisté, pour certaines maternités, à accepter les accompagnant.e.s dans les services de suites de naissances dès le mois de mars, alors même que le CNGOF ne le préconise qu'à partir du 27 avril. L'autorisation de la présence de l'accompagnant.e s'accompagne alors de mesures de confinement : " afin de limiter les circulations dans la maternité, la présence du père sera autorisée pour la naissance et jusqu'à $24 \mathrm{~h}$ après l'accouchement en confinement strict dans la chambre. Toute sortie de la chambre sera considérée comme définitive. » (CHU Lille, 24 mars 2020)

\section{Disparités territoriales et parcours de soins}

L'analyse des pratiques discursives indique que les mesures prises en milieu hospitalier quant à la présence de l'accompagnant.e ont été du ressort de chaque maternité et qu'elles ont été prises d'une part en s'adaptant au contexte local en termes d'évolution épidémiologique (CNGOF, 30 mars 2020) ${ }^{19}$ (CNSF et al., 23 mars 2020) ${ }^{20}$ et d'autre part en fonction des ressources locales matérielles et humaines permettant le respect ou non des mesures barrières et la préservation d'un nombre suffisant de professionnels de la naissance en exercice (Ouest France, 2 avril 2020) ${ }^{21}$ (CNOSF et al., 27 mars 2020) ${ }^{22}$. Si les décisions ont été du ressort de chaque maternité, une certaine coordination entre les maternités a pu se mettre en place au sein d'un même territoire : «(...) l'ARS (Agence Régionale de Santé) Bourgogne-Franche-Comté a annoncé que toutes les maternités de la région s'étaient mises d'accord sur une organisation commune : Les papas peuvent assister à la dernière phase de l'accouchement (...) rester pendant 2 heures avec la maman et le nouveau-né (...) et ne pourront revenir que lors de la sortie de leur conjointe et du bébé. » (France 3 Bourgogne-Franche-Comté, 2 avril 2020). Des disparités de mesures semblent cependant aussi avoir pu co-exister au sein de mêmes territoires sanitaires (TV5 Monde, 31 mars 2020) ${ }^{23}$. On peut alors se demander si ces disparités au sein d'un même territoire ont été susceptibles d'avoir modifié les parcours de soins des femmes enceintes, celles-ci pouvant privilégier un accouchement dans une maternité ayant des mesures d'éviction des accompagnant.e.s moins restrictives. Les mesures restrictives au sein des maternités semblent par ailleurs avoir participé à amener davantage de femmes et de couples à envisager un accouchement à domicile. L'Association Professionnelle de l'Accouchement Accompagné à Domicile (APAAD) et l'Association Nationale des Sages-Femmes Libérales (ANSFL) ont annoncé une hausse exponentielle de la demande d'accouchements à domicile "du fait de l'interdiction des personnes accompagnantes dans les maternités et de la limitation de leur projet de naissance » (APAAD, ANSFL, 25 mars 2020). La presse s'est également fait l'écho de cette recrudescence de projets d'accouchements à domicile pendant le confinement, recrudescence observée non seulement en France (France Soir, 31 mars 2020) ${ }^{24}$ mais également dans d'autres pays comme la Suisse (RTS, 25 mars 2020) ${ }^{25}$, le Canada (Beaudoin, D., 17 mai 2020) ${ }^{26}$ ou encore les Etats Unis (AFP, 8 avril 2020) ${ }^{27}$. A ce jour, aucune donnée factuelle ne 
permet cependant de dire si ces projets d'accouchements à domicile ont abouti et s'il y a eu effectivement davantage d'accouchements à domicile durant le confinement.

\title{
Acceptabilité sociale des mesures d'éviction des accompagnant.e.s
}

Nous avons analysé des écrits publiés pendant la période de la Covid 19 qui pouvaient refléter le degré d'acceptabilité sociale des mesures d'éviction des accompagnant.e.s autour de la naissance d'une part et des modèles décisionnels ayant participé à l'application de ces mesures d'autre part. Nous présentons un échantillon de ces écrits ici.

La Conférence Internationale des Sages-Femmes déclare en mars 2020

\begin{abstract}
" s'inquiéter de voir que les droits fondamentaux des femmes, de leurs bébés et de leurs sages-femmes soient violés par l'introduction, dans de nombreux pays, de protocoles inappropriés pour la gestion des grossesses, des accouchements et des soins postnatals en réponse à la pandémie de covid-19. Ces protocoles inappropriés ne sont pas basés sur des preuves fiables et sont nuisibles pour les femmes et leurs bébés. (...) il est essentiel que les protocoles de grossesse et d'accouchement pendant la pandémie de coronavirus soient fondés sur des preuves et respectent les droits fondamentaux de toutes les femmes et de leurs nouveau-nés. (...) le soutien continu d'un partenaire de naissance augmente l'accouchement vaginal spontané, raccourcit le travail et diminue les naissances par césarienne et autres interventions médicales. » (ICM, Mars 2020)
\end{abstract}

Le Collectif «Stop aux violences obstétricales et gynécologiques » a mis en ligne le 26 mars 2020, une pétition réclamant au ministre de la santé " la rédaction de recommandations nationales issues de la concertation entre les différents acteurs de la filière de soins de périnatalité afin de garantir aux femmes la présence de la personne de leur choix en salle de naissance ${ }^{28}$.

Le 27 mars, la cellule de crise " Covid19-sage-femme ", dans une lettre ouverte au gouvernement, déclare que " la place et le rôle indispensable du conjoint lors de l'accouchement, soulignés et réaffirmés par l'OMS dans le contexte de crise, sont aujourd'hui remis en cause » et demande que soit garantie " une prise en charge "décente" et humaine des femmes enceintes, des parturientes, des parents et de leurs enfants ; limitant les séquelles physiques, psychiques et émotionnelles ». (CNOSF et al., 27 mars 2020)

Le 3 avril 2020, quatre associations françaises de sages-femmes ${ }^{29}$ publient un communiqué commun dans lequel elles déclarent que

\footnotetext{
« les femmes sont confrontées à une solitude déchirante pendant l'accouchement et en post-partum. Ce sentiment s'ajoute à l'état de grande vulnérabilité due aux remaniements psychiques et hormonaux physiologiques, inhérents au bon déroulement de la grossesse et de l'accouchement et font craindre une majoration des dépressions du post partum ». (ANSFT et al., 3 avril 2020)
}

Des chercheures de l'Institut National des Etudes Démographiques ont affirmé que « ce refus de la présence d'un soutien continu pendant l'accouchement porte atteinte aux droits reproductifs et peut entraîner des conséquences sur la santé physique, psychique et sociale ». (Mazuy, M. et al., 6 mai 2020)

Le collectif Tou.te.s Contre les Violences Obstétricales et Gynécologiques a mis en ligne une carte des violences obstétricales en période de Covid-19 où, à partir d'une « enquête nationale sur la naissance pendant la pandémie de Covid 19 ", sont notamment référencés différents témoignages de femmes ayant vécu l'éviction de l'accompagnant.e en maternité comme une violence obstétricale. (Sogny, A., Juin 2020) (Tou.te.s Contre les Violences Obstétricales et Gynécologiques, Juillet 2020)

Le CNGOF, dans son communiqué du 30 mars, écrit avoir reçu pour les recommandations " l'entière adhésion du Collectif Interassociatif Autour de la Naissance (CIANE) ${ }^{30}$ » c'est-à-dire l'adhésion des représentants des usagers. Or, le 
lendemain de cette publication, le CIANE publiait un démenti : « le CIANE n'a à aucun moment été consulté ni n'a exprimé un avis sur la question (...). Nous ne nous sommes donc pas prononcées en faveur de tel ou tel protocole, et certainement pas en faveur de l'absence du père. » (CIANE, 31 mars 2020) ${ }^{31}$ Par ailleurs, le CNGOF n'indique pas dans son communiqué s'être concerté avec les autres professionnels de la naissance pour émettre ses recommandations.

La perception d'une verticalité des prises de décisions autour de la naissance en temps de Covid 19 s'étend à des autorités en santé autres que le CNGOF. La cellule de crise « Covid19-sage-femme », regroupant la majorité des organisations professionnelles des sages-femmes, affirme ainsi dans un communiqué sa perception du manque de prise en considération de « l'avis des professionnels de terrain » de la part de la Haute Autorité en Santé déclarant que les réponses rapides dans le cadre de la pandémie de Covid 19 " ignorent les avis des professionnels de terrain » (Cellule de crise " Covid19-sagefemme », 7 avril 2020) ${ }^{32}$. Les sages-femmes ont déclaré par ailleurs que les femmes et leurs accompagnant.e.s ainsi que les sages-femmes ont été insuffisamment pris.e.s en compte par le gouvernement lors de la dotation en matériel de protection et que cela a participé à l'éviction des accompagnant.e.s. Ils.Elles en ont référé au gouvernement le 27 mars dans une "lettre ouverte des sages-femmes à Monsieur le Ministre des Solidarités et de la Santé et Monsieur le Directeur Général de la Santé » (CNOSF et al., 27 mars 2020)33.34

Réalisant $87,4 \%$ des accouchements par voie basse non instrumentale et étant parmi les principaux professionnels responsables de la surveillance de la grossesse et de la préparation à la naissance et à la parentalité (INSERM, DREES, 2017), les sagesfemmes ont été parmi les professionnel.le.s de terrain les plus touché.e.s dans leurs pratiques par l'ensemble des recommandations autour de la naissance. Or, l'analyse des pratiques discursives indique ainsi que l'élaboration des recommandations et des décisions prises autour de la naissance pendant le confinement semblent avoir été faites sans consultation participative suffisante de l'ensemble des sages-femmes ouvrant au quotidien dans les services des maternités et en médecine de ville.

L'analyse de contenu des textes publiés pendant la période du Covid 19 montre une difficile acceptabilité sociale non seulement des mesures d'éviction des accompagnant.e.s autour de la naissance mais également des modèles décisionnels ayant participé à l'application des mesures autour de la naissance. En effet, notre analyse de contenu de ces textes met en exergue que les mesures d'éviction des accompagnant.e.s autour de la naissance semblent pouvoir être perçues comme issues de modèles décisionnels verticaux ou tout au moins insuffisamment participatifs. Qu'elles semblent pouvoir être perçues comme issues principalement de modèles hygiénistes biomédicaux et prenant insuffisamment en considération l'ensemble des enjeux liés à la présence de l'accompagnant.e. Qu'elles semblent pouvoir être perçues comme insuffisamment argumentées sur le plan scientifique et comme sous-estimant l'ensemble des effets qui en découlent sur la santé des nouveau-nés, des femmes et plus globalement des futurs parents et de la fratrie. Qu'elles semblent enfin pouvoir être perçues comme portant atteinte aux droits sexuels et reproductifs des femmes et ne répondant pas aux quatre principes fondamentaux de l'éthique médicale (autonomie, bienfaisance, non malfaisance, justice).

\section{Conclusion}

Il se dégage de l'analyse des pratiques discursives autour de la naissance pendant le confinement un ensemble de faits anthropologiques. Un recul majeur du droit des femmes à être accompagnées de la personne de leur choix lors de leur grossesse, de leur accouchement et des suites de la naissance de leur.s nouveau-né.s (droit réaffirmé par l'OMS le 18 mars 2020) a été observé. Les pratiques issues des discours ont de plus entravé l'accès des futurs parents à une préparation à la naissance et à la parentalité (préconisations du Plan Périnatalité 2005-2007). Elles ont porté atteinte à la sécurité émotionnelle des femmes enceintes (Bohren, M.A.et al., 2017) qui est un objectif 
primordial du dernier Plan Périnatalité 2005-2007 (Molénat, F., 2007). Les discours hétéronormés autour de la naissance ont pu invisibiliser la diversité des modèles du couple, de la famille et des choix des femmes quant à leur accompagnant.e autour de la naissance. Par ailleurs, la mise en ouvre d'approches participatives, telles que préconisées pour l'élaboration des politiques périnatales (Puech, F., 2007) a été insuffisante, les modèles décisionnels étant vécus par les sages-femmes et les usagers comme verticaux 35 . Enfin, les mesures d'éviction des accompagnant.e.s ont globalement été perçues comme insuffisamment argumentées sur le plan scientifique, ne prenant pas suffisamment en considération l'ensemble des enjeux psychologiques liés à la présence de l'accompagnant.e autour de la naissance. Ce dispositif de restriction aurait ainsi sous-estimé l'ensemble des effets de cette éviction sur la santé des nouveau-nés, des femmes et plus globalement des futurs parents et de la fratrie et n'aurait pas appliqué les quatre principes fondamentaux de l'éthique médicale (autonomie, bienfaisance, non malfaisance, et responsabilité). Ce que nous apprend ici la pandémie " de nos sociétés, de nos politiques de santé publique, des souverainetés épistémologiques de la recherche et des praxis individuelles et collectives » (Descarpentries, J. et al., 2020), c'est l'absence d'application de modèles réellement participatifs en santé génésique, c'est la prédominance de la gouvernance des corps et de la soumission des femmes par des modèles décisionnels verticaux issus de modèles hygiénistes biomédicaux. La pandémie nous montre ici la nécessité d'une vigilance constante face au risque majeur du recul de droits fondamentaux " dans ce monde incertain, soumis à l'autorité des politiques de gestion d'un virus » (Descarpentries, J. et al., 2020). L'analyse des pratiques discursives autour de la naissance lors de la pandémie nous rappelle que le respect de l'application des droits des femmes nécessite une vigilance accrue en situations de crises, que celles-ci soient économiques, sociales, politiques ou sanitaires.

Mettant en évidence que les mesures restrictives autour de la naissance ont été vécues comme insuffisamment argumentées sur le plan scientifique et comme issues de modèles décisionnels verticaux, ce travail débouche sur au moins deux questions épistémologiques majeures en santé génésique, questions qui ont sous-tendu non seulement les discours sur les pratiques mais également la pratique issue des discours.

La première question épistémologique majeure qui est posée par les résultats de notre recherche est ainsi relative à une élaboration perçue comme insuffisamment participative des politiques périnatales pendant le confinement. L'étude de différents courants de sciences participatives ou collaboratives permet de comprendre non seulement les enjeux scientifiques des approches participatives mais également leurs enjeux sociaux et politiques et d'envisager la recherche-action participative ou collaborative autour de la naissance comme un outil de promotion de la santé émancipatrice (Chapela, M., 2008). En 1994, Silvio O. Funtowicz et Jerome R. Ravetz proposent une remise en question de ce qu'ils nomment les « approches traditionnelles scientifiques " pour penser une " science post-normale de l'incertitude et de la complexité » tout en plaidant pour une approche "systémique, synthétique et humaniste " prenant en compte une "pluralité de perspectives ou points de vue légitimes ». Ils appellent à la démocratisation de l'expertise permettant aux populations, à la société civile, à une " communauté élargie de pairs » (intégrant une pluralité de savoirs d'épistémologies divers à travers la participation de personnes traditionnellement non reconnues comme experts), d'être parties prenantes dans les recherches menant directement ou indirectement à des décisions politiques les concernant. La science post-normale peut être considérée comme proposant une prise en compte épistémologique plurielle nécessaire lorsque « les faits sont incertains, les valeurs sont polémiques, les enjeux sont importants et les décisions sont urgentes » (Funtowicz, S., Ravetz, J., 1994). En 2001, Michel Callon, Pierre Lascoumes et Yannick Barthe, dans un essai sur la démocratie technique, vont émettre des propositions, théoriques et pratiques, à propos de l'organisation de "forums hybrides », espaces publics ouverts permettant la délibération et la controverse sur des choix techniques collectifs entre " experts » et «profanes », pour " agir dans un monde incertain » de manière plus qualitative et efficiente (Callon, M. et al., 2001). Ces approches prennent en considération l'urgence de penser des modèles collaboratifs de recherche permettant 
une co-construction de la recherche avec les groupes sociaux confrontés en premier chef à ces questions, dans une optique de recherche de justice sociale et cognitive (Sousa Santos, B., 2016). Pour Baoventura de Sousa Santos, il s'agit également de porter l'attention sur les disparités de reconnaissance épistémologique. Il concentre ses recherches sur la proposition d'une autre science " pluriverselle » (Dufoix, S. et al., 2019) prenant en considération les différentes épistémologies co-existant. Les approches participatives sont en effet des voies intéressantes à explorer pour renouveler les épistémologies en santé génésique. Il serait cependant simpliste et naïf de penser qu'une approche participative permettant à chacune des parties prenantes de pouvoir s'exprimer conduirait systématiquement à des consensus (Massé, R., 2012). Il s'agirait également de se méfier d'une confusion entre " populisme méthodologique » et «populisme idéologique » (Chauveau, J.P., 1994).

La deuxième question épistémologique majeure qui est posée est relative à l'objectivité scientifique affichée, mais non socialement perçue, des mesures restrictives autour de la naissance. L'usage social et politique des résultats de notre recherche invite d'une part à une mise en lumière des dissonances entre la « base morale " $3^{6}$ affichée des décisions scientifiques et les différentes réalités qui s'entremêlent dans les fils de ces décisions. D'autre part, il interpelle sur les choix épistémologiques en santé génésique ou, dit autrement, sur les choix de modèles de construction des savoirs et de modèles décisionnels issus de la construction de ces savoirs. Rappelons, notamment, que l'objectivité des sciences prônée par le cercle de Vienne a été réfutée par l'école de Francfort et ses héritiers qui ont démasqué la " raison instrumentale »37. Habermas invite à être vigilant quant à " l'illusion objectiviste, cette illusion des sciences selon laquelle il y aurait un en-soi de faits déjà structurés en lois et qui masque la constitution de ces mêmes faits, de sorte que n'apparait plus combien la connaissance et les intérêts du monde vécu sont entremêlés » (Habermas, J., 1973 : 141). Popper interroge quant à lui l'objectivité de la science en affirmant qu' " une décision morale n'a jamais de racine scientifique. En revanche, une décision scientifique a toujours une base morale » (Baudoin, J., 1989). Il interpelle également quant aux choix épistémologiques : « ce choix n'est pas seulement affaire d'opinion personnelle ou de position intellectuelle. C'est une décision d'ordre moral, pouvant avoir une profonde influence sur notre attitude envers les autres hommes et les problèmes de la société » (Popper, K., 1979 : 158).

\section{Bibliographie}

Agence France Presse. (8 avril 2020). Angoissées par le coronavirus, des Américaines accouchent à domicile. [En ligne] https://www.loophaiti.com/content/angoissees-par-le-coronavirus-desamericaines-accouchent-domicile (consulté le 1 juin 2020)

ANSFT et al. (3 avril 2020). Quatre associations nationales de sages-femmes s'expriment sur la prise en charge des femmes à " 17 jours de confinement dû au coronavirus ». [En ligne] https://ordresagesfemmes31.fr/wp-content/uploads/2020/o4/Texte-COVID-20_03_29-v11-etlogo.pdf (consulté le 7 avril 2020)

APAAD, ANSFL. (25 mars 2020). Accouchement à domicile dans le cadre de l'épidémie Covid-19. 25 mars 2020. [En ligne] https://www.apaad.fr/accouchement-a-domicile-dans-le-cadre-delepidemie-covid-19/ (consulté le 30 mai 2020)

Bardin, L. (2013). L'analyse de contenu. Paris : Presses Universitaires de France DOI : 10.3917/puf.bard.2013.01

Baudoin, J. (1989). Karl Popper. Que Sais-je ? Paris : Presses Universitaires de France, p 51

Beaudoin, D. (17 mai 2020). Avec la pandémie, les sages-femmes plus sollicitées que jamais. Radio-canada. 17 mai 2020. [En ligne] https://ici.radio-canada.ca/nouvelle/1703070/avec-lapandemie-les-sages-femmes-plus-sollicitees-que-jamais (consulté le 1 juin 2020)

Bohren, M.A., Hofmeyr, G., Sakala, C., Fukuzawa, R.K., Cuthbert, A. (2017). Continuous support for women during childbirth. Cochrane Database of Systematic Reviews, Issue 7. Art. No. : CDoo3766

Callon, M., Lascoumes, P. et Barthe,Y. (2001). Agir dans un monde incertain. Essai sur la démocratie technique, Paris : Seuil

Cellule de crise « Covid19-sage-femme ». (7 avril 2020). Communiqué aux sages-femmes du 07 avril 2020. [En ligne] https://www.onssf.org/wp-content/uploads/2020/o3/Communiqué-aux- 
SF_07042020_vd2.pdf (consulté le 9 avril 2020)

CFEF, CNGOF. (Mars 2020). Recommandations pour la pratique des échographies obstétricales et fotales pendant la période stade 3 de l'épidémie COVID-19. Mars 2020 [En ligne] https://sfipradiopediatrie.org/wp-content/uploads/2020/03/Recommandations-CFEF-CNGOF-002.pdf (consulté le 31 mars 2020)

Chapela, M. (2008). Promoción de la salud. Un instrumento del poder y una alternativa emancipatoria. University of New Mexico. UNM Digital Repository [En ligne] https://digitalrepository.unm.edu/lasm_cucs_es/16 (consulté le 1 mai 2020)

Chauveau, J.P. (1994). Participation paysanne et populisme bureaucratique. Essai d'histoire et de sociologie de la culture du développement, in : Jacob, J.P., Delville, P. (dir.) Les associations paysannes en Afrique : organisation et dynamiques, Paris, APAD-Karthala-IUED, p 25-60

CHU Lille. (24 mars 2020). Coronavirus. Consignes aux accompagnants. [En ligne] https://www.chu-lille.fr/wp-content/uploads/2020/o3/Covid-19-Affiche-maternite \%CC \%81240320.pdf (consulté le 15 avril 2020)

CHU Rouen. Maternité Charles Nicolle. (31 mars 2020). Covid-19 : Présence du père à l'accouchement. 31 mars 2020. [En ligne] https://maternite.chu-rouen.fr/covid-19-presence-dupere-a-laccouchement/ (consulté le 15 avril 2020)

CIANE. (31 mars 2020). Mise au point du Ciane. [En ligne] https://ciane.net/2020/o3/mise-aupoint-du-ciane/ (consulté le 15 mai 2020)

CNGOF. (27 mars 2020). Assistance des accompagnants à l'accouchement. 27 mars 2020. [En ligne] https://www.reseaux-perinat-hn.com/wp-content/uploads/2020/04/200327Accompagnant_SDN_CNGOF.pdf (consulté le 1 avril 2020)

CNGOF, SYNGOF. (30 mars 2020). COVID-19 : Recommandations du CNGOF et du SYNGOF pour le suivi de grossesse et l'accouchement. 30 mars 2020 [En ligne] https://syngof.fr/communiques-presse/covid-19-recommandations-du-cngof-et-du-syngofpour-le-suivi-de-grossesse-et-laccouchement/ (consulté le 30 mars 2020). 38

CNGOF. (27 avril 2020). Position du CNGOF sur la présence d'un accompagnant en maternité dans le contexte de Covid-19. 27 avril 2020 [En ligne] www.cngof.fr/coronavirus-gocngof/apercu ?path =COVID-accompagnants-accouchement-2.pdf (consulté le 28 avril 2020)

CNOSF et al. (27 mars 2020). Lettre ouverte à Monsieur le Ministre des Solidarités et de la Santé et Monsieur le Directeur Général de la Santé. 27 mars 2020. [En ligne] https://www.onssf.org/wp-content/uploads/2020/o3/Lettre-ouverte-au-ministre-des-SF-du27-Mars-2020.pdf (consulté le 30 avril 2020)

CNSF et al. (23 mars 2020). Covid-19 - Communiqué de la Cellule de Crise Sage-Femme. Lundi 23 mars. [En ligne] https://www.cnsf.asso.fr/communique-de-la-cellule-de-crise-sage-femmelundi-23-mars/ (consulté le 15 avril 2020)

Collectif Stop Aux Violences Obstétricales et Gynécologiques. (26 mars 2020). Pétition "FR Garantir la présence de l'accompagnant en salle de naissance malgré le Covid19". [En ligne] https://www.change.org/p/olivier-veran-ministre-de-la-santé-fr-garantir-la-presence-de-laccompagnant-en-salle-de-naissance-malgre-le-covid-19 (consulté le 1 juin 2020)

Descarpentries J., Andrieu B., (2020). Appel à contribution : que nous apprend la pandémie ? Numéro HS Recherches et Educations. [En ligne] https://journals.openedition.org/rechercheseducations/ (consulté le 30 avril 2020)

De Sousa Santos, B. (2016). Epistémologies du Sud. Mouvements citoyens et polémique sur la science. Paris : Desclée de Brouwer

De Sousa Santos, B. (2018). O fim do império cognitivo. Editions Almedina

Dufoix, S., Macé, E. (2019). Les enjeux d'une sociologie mondiale non hégémonique. Editions du Croquant «Zilsel ». 2019/1 N 5, p 88-121

Evrard N. (1er avril 2020). Suivi de grossesse et accouchement : les gynécos fixent les règles en période épidémique. Le Généraliste. 1er avril 2020. [En ligne] https://www.legeneraliste.fr/actualites/article/2020/04/o1/suivi-de-grossesse-etaccouchement-les-gynecos-fixent-les-regles-en-periode-epidemique_322092 (consulté le 28/05/2020)

Foucault, M. (1977). Dits et écrits, volume III, Paris : Gallimard, p 299-300

France 3 Bourgogne-Franche-Comté. (2 avril 2020). Coronavirus Covid-19 : est-ce que la présence des papas est autorisée dans les maternités pendant l'accouchement ? [En ligne] https://france3-regions.francetvinfo.fr/bourgogne-franche-comte/cote-d-or/coronavirus-covid19-est-ce-que-presence-papas-est-autorisee-maternites-accouchement-1805752.html (consulté le 1 mai 2020)

Funtowicz, S., Ravetz, J. (1994). Uncertainty, complexity and post-normal science. Environmental Toxicology and Chemistry, vol. 13, No. 12, p 1881-1885

Gélis, J. (1988). La sage-femme et le médecin. Une nouvelle conception de la vie. Paris : Fayard.

Habermas, J. (1973). La technique et la science comme idéologie. Trad. J-R Ladmiral. Gallimard, p 141 
INSERM, DREES. (2017). Enquête nationale périnatale. Rapport 2016. Les naissances et les établissements, situation et évolutions depuis 2000.

International Confederation of Midwives. (Mars 2020). Les droits des femmes doivent être respectés lors de l'accouchement durant la pandémie de coronavirus. [En ligne] https://www.internationalmidwives.org/assets/files/news-files/2020/03/french-statement.pdf . (consulté le 18 avril 2020)

Knibiehler, Y. (2016). Chapitre 2. L'accouchement : affaire publique, affaire de médecins. Dans : Y. Knibiehler, Accoucher : Femmes, sages-femmes et médecins depuis le milieu du XXe siècle (p 35-52). Rennes, France : Presses de l'EHESP.

Laufer S. (Mars 2020). Ne pas interdire l'accès aux salles d'accouchement aux futurs papas dû au Covid-19. [En ligne] https://www.change.org/p/chuv-ne-pas-interdire-l-accès-aux-salles-daccouchement-aux-futurs-papas-dû-au-covid-19 (consulté en ligne le 3 mai 2020)

Massé, R. (2012). Expertises éthiques savantes et profanes en santé publique : défis et enjeux pour une éthique de la discussion.S.F.S.P. « Santé Publique » 2012/1 vol. 24, p 49-61 DOI : $10.3917 /$ spub.121.0049

Mazuy, M. et al. (INED). (6 mai 2020). IVG, grossesse, accouchement : quand la crise sanitaire menace les droits des femmes. The conversation. [En ligne] https://theconversation.com/ivggrossesse-accouchement-quand-la-crise-sanitaire-menace-les-droits-des-femmes-137625 (consulté le 7 mai 2020)

Ministère de la Solidarité de la Santé et de la Protection Sociale. (2004). Plan "Périnatalité" 2005-2007 : humanité, proximité, sécurité, qualité. Paris : Ministère chargé de la santé

Molénat, F. (2007). Le tournant du Plan Périnatalité 2005-2007. Contraste, 26(1), p 127-135 DOI : 10.3917/cont.026.0127

Morel M.F., Gélis J. (1991). La sage-femme ou le médecin. Une nouvelle conception de la vie. In : Annales. Economies, sociétés, civilisations. 46 année, N. 3, 1991, p 636-640

Morel, M.F. (2008). Histoire de la naissance en France (xviie-xxe siècle). ADSP n ${ }^{\circ} 61 / 62$, p 22-28

ONSSF. (Juin 2020). Ségur de la Santé. Contribution de l'organisation nationale syndicale des sages-femmes. [En ligne] https://www.onssf.org/wpcontent/uploads/2020/06/202006_SEGUR_ONSSF.pdf (consulté le 1 août 2020)

Ouest France. (2 avril 2020). Coronavirus. La polyclinique de Nancy autorise de nouveau les accompagnants en salle d'accouchement. [En ligne] https://www.ouestfrance.fr/sante/virus/coronavirus/coronavirus-la-polyclinique-de-nancy-autorise-de-nouveaules-accompagnants-en-salle-d-accouchement-6799416 (consulté le 3 avril 2020)

Popper, K. (1979). La société ouverte et ses ennemis, tome 1, L'ascendant de Platon, tome 2, Hegel et Marx. Paris : Editions du Seuil, p 158

Puech, F., Pauchet-Traversat, A.F., Patureau, J. (2007). L'organisation autour de la grossesse et de la naissance. ADSP 35-57

Raso, A. (31 juillet 2020). Ségur de la santé : "oubliées et méprisées", les sages-femmes de l'hôpital de Haguenau se mobilisent. [En ligne] https://www.francebleu.fr/infos/economiesocial/segur-de-la-sante-oubliees-et-meprisees-les-sages-femmes-de-l-hopital-de-haguenau-semobilisent-1596034807 (consulté le 1 août 2020)

Rousseau, A., Baumann, S., Gaucher, L. (2020). Rapport Enquête Covid-SF. UVSQ, CNSF

RTS. (25 mars 2020). Hausse des accouchements à domicile en raison de la pandémie de Covid19. [En ligne] https://www.rts.ch/info/suisse/11191496-hausse-des-accouchements-a-domicileen-raison-de-la-pandemie-de-covid-19.html (consulté le 3 juin 2020)

Sogny, A. (Juin 2020). Un collectif publie une carte des violences obstétricales en période de Covid-19. [En ligne] https://www.marieclaire.fr/enquete-nationale-accouchement-covid-19 (consulté le 20 juin 2020)

Tou.te.s Contre les Violences Obstétricales et Gynécologiques. (Juillet 2020). Rapport d'enquête sur la grossesse, l'accouchement et le post-partum pendant l'épidémie de Covid-19. [En ligne] http://stop-vog.fr/rapport_enquete_naissance_covid.pdf (consulté le 31 juillet 2020)

TV5Monde. Terriennes. (31 mars 2020). Enceinte à l'heure du Covid-19 : les gynécologues appellent à assouplir les règles de confinement. [En ligne] https://information.tv5monde.com/terriennes/enceinte-l-heure-du-covid-19-les-gynecologuesappellent-assouplir-les-regles-de (consulté le 20 avril 2020)

World Health Organization (Éd.). (2016). Recommendations on antenatal care for a positive pregnancy experience. Geneva : World Health Organization

World Health Organization. (2018). Recommendations on intrapartum care for a positive childbirth experience. S.l. : World Health Organization

World Health Organization. (2020). Questions-réponses sur la COVID-19, la grossesse, l'accouchement et l'allaitement. [En ligne] https://www.who.int/fr/news-room/q-a-detail/q-aon-covid-19-pregnancy-and-childbirth (consulté le 18 mars 2020) 


\section{Notes}

1 « Par dispositif, j'entends une sorte - disons - de formation qui à un moment donné a eu pour fonction majeure de répondre à une urgence. Le dispositif a donc une fonction stratégique dominante...

J'ai dit que le dispositif était de nature essentiellement stratégique, ce qui suppose qu'il s'agit là d'une certaine manipulation de rapports de force, d'une intervention rationnelle et concertée dans ces rapports de force, soit pour les développer dans telle direction, soit pour les bloquer, ou pour les stabiliser, les utiliser. Le dispositif donc est toujours inscrit dans un jeu de pouvoir, mais toujours lié aussi à une ou à des bornes de savoir, qui en naissent, mais, tout autant, le conditionnent. C'est ça le dispositif : des stratégies de rapports de force supportant des types de savoir, et supportés par eux ». (Foucault, M., 1994 : 299, 300)

2 A noter que nous faisons référence au communiqué publié le 30 mars et non au contenu modifié courant juin mais toujours référencé à la date du 30 mars sur le site du SYNGOF.

3 Cette restriction pour les échographies est reprise dans un communiqué du Collège Français d'Echographie Fœtale (CFEF) et du Collège National des Gynécologues et Obstétriciens Français (CNGOF) publié en mars et précisant « Aucun accompagnant avec la femme enceinte, aussi bien dans le cabinet de consultation que dans la salle d'attente ». (CFEF, CNGOF, 30 mars 2020)

4 « En suites de couches, dans la mesure où les durées de séjour ont été raccourcies, il semble préférable d'envisager une absence totale de sortie de l'accompagnant qui reste auprès de la mère. Si le séjour est plus long ou que l'accompagnant ne peut rester dormir sur place, une seule venue est autorisée par jour, toute sortie est considérée comme définitive et seul un retour le lendemain peut être autorisé (...). L'accompagnant s'engage à rester « confiné » pendant toute la durée de sa présence afin d'éviter au maximum les allées et venues (...). » (CNGOF, 27 avril 2020)

5 Les conditions de cette présence sont émises dont la recherche de signes cliniques chez la mère et l'accompagnant, l'engagement au respect des gestes barrières...

6 D'autres textes publiés durant le confinement par des organisations professionnelles, des collectifs d'usagers ou des journalistes ont pu montrer ce même usage de mots restrictifs évoquant une vision normative et hétérosexuelle du couple et de la famille.

7 «Un groupe de travail dirigé par Anne Rousseau de l’Université de Versailles-Saint-Quentinen-Yvelines en collaboration avec le groupe Accord (Assembler, Coordonner, Comprendre, Rechercher, Débattre en Soins Primaires) et le Collège National des Sages-Femmes de France, société savante des sages-femmes, a mené une étude de cohorte par auto-questionnaire en ligne du 29/04/2020 au 15/05/2020 auprès des sages-femmes libérales, territoriales, hospitalières et coordinatrices (...) visant à décrire l'adaptation des pratiques et d'étudier plus précisément la mise en place des téléconsultations. » (Rousseau, A. et al., 2020)

8 Le collectif Tou.te.s Contre les Violences Obstétricales et Gynécologiques a mené une enquête par auto-questionnaire en ligne du 6 mai au 31 mai 2020 auprès de femmes et "visait les accouchements qui se sont déroulés entre le 15 février (date de l'annonce par l'ancienne ministre de la santé Mme Agnès Buzin du premier décès dû au Covid-19 sur le sol français) et le 31 mai 2020. (...) 2727 réponses reçues, parmi lesquelles environ 2000 témoignages. » (Tou.te.s Contre les Violences Obstétricales et Gynécologiques., Juillet 2020)

9 Les résultats tirés de l'analyse des textes publiés durant le confinement sont confirmés par ces résultats de l'enquête Covid-SF : 44,3 \% des sages-femmes libéraux.ales et 32,8 \% des sagesfemmes territoriaux.ales ayant répondu à l'auto-questionnaire ont déclaré avoir annulé ou reporté les séances collectives de préparation à la naissance. A noter que les séances individuelles ont été annulées ou reportées pour $10.7 \%$ des sages-femmes libéraux.ales et $20.7 \%$ des sagesfemmes territoriaux.ale interrogé.e.s.

Si le rapport d'enquête n'aborde pas cette question pour les sages-femmes hospitaliers.ères, notre analyse du contenu de communiqués d'organisations professionnelles et d'associations d'usagers, de sites internet des maternités et d'articles journalistiques publiés durant le confinement montre que les maternités ont, de façon très généralisée, également annulé des séances collectives de préparation à la naissance, y compris des visites des maternités pour les femmes et leurs accompagnant.e.s.

10 Les résultats tirés de l'analyse des textes publiés durant le confinement sont confirmés par ces résultats de l'enquête Covid-SF : 88,5 \% des sages-femmes libéraux.ales et 66,4 \% des sagesfemmes territoriaux.ales ayant répondu à l'auto-questionnaire ont réalisé des téléconsultations durant la période de l'enquête (dont suivis de grossesse et préparation à la naissance et à la parentalité). Le rapport d'enquête n'aborde pas cette question pour les sages-femmes hospitaliers.ères.

11 Les résultats tirés de l'analyse des textes publiés durant le confinement sont confirmés par ces résultats de l'enquête Covid-SF : « les pères ne sont pas autorisés en consultation ou en cours de préparation à la naissance pour $77,8 \%$ des sages-femmes libérales ayant répondu (...), pour $85,3 \%$ des sages-femmes territoriales ayant répondu » et les accompagnant.e.s ne sont pas autorisé.e.s en consultation pour 87,6 \% des sages-femmes hospitaliers.ères ayant répondu.

12 Les résultats tirés de l'analyse des textes publiés durant le confinement sont confirmés par ces résultats de l'enquête Covid-SF : 71,2 \% des sages-femmes hospitaliers.ères et 53,1 \% des sages- 
femmes coordinateurs.trices ayant répondu déclarent que les accompagnant.e.s n'étaient « pas autorisé.e.s en service de Grossesse à Haut Risque ».

13 L'analyse de contenu de publications dans la presse, de témoignages de parents sur des blogs et des sites internet des maternités semble indiquer que durant les premiers jours du confinement, de nombreuses maternités ont totalement interdit l'accès des accompagnant.e.s en salles de naissances. Ensuite, les protocoles semblent s'être assouplis pour la phase active du travail, lors de l'accouchement et durant les deux heures suivant la naissance, tout en pouvant garder une interdiction des accompagnant.e.s pendant la première phase de travail. Exemple : " La polyclinique Majorelle, située à Nancy (Meurthe-et-Moselle), autorise de nouveau la présence du conjoint lors d'un accouchement. Cela n'était plus possible depuis le 23 mars dernier, à cause de l'épidémie de coronavirus. La clinique l'a annoncé jeudi 2 avril dans un communiqué de presse, indique France 3 Grand-Est. Une décision qui fait suite aux recommandations publiées par le Collège national des gynécologues et obstétriciens, le 27 mars dernier. Pour pallier l'absence de l'accompagnant, la clinique proposait de suivre les accouchements en vidéo, via des applications comme Whatsapp ou FaceTime. » (Ouest France, 2 avril 2020)

L'enquête Covid-SF ne précise pas dans ses résultats les variations temporelles relatives à cette question. Les résultats montrent uniquement qu'à la période où le questionnaire a été mis en ligne (autour de la période finale du confinement, du 29/04/2020 au 15/05/2020) 6,4 \% des sages-femmes hospitaliers.ères et $3,1 \%$ des sages-femmes coordinateurs.trices déclarent que l'accompagnant.e n'est pas du tout autorisé.e en salles de naissances dans la maternité où ils.elles exercent.

Pour l'enquête réalisée par le collectif Tou.te.s Contre les Violences Obstétricales et Gynécologiques, $11 \%$ des femmes ayant répondu déclarent que la présence de l'accompagnant.e. était totalement interdite au moment de l'accouchement. (Tou.te.s Contre les Violences Obstétricales et Gynécologiques, Juillet 2020)

14 Les résultats tirés de l'analyse des textes publiés durant le confinement sont confirmés par ces résultats de l'enquête Covid-SF : 74,7 \% des sages-femmes hospitaliers.ères et 59,4 \% des sagesfemmes coordinateurs.trices ayant répondu déclarent que l'accompagnant.e n'est pas autorisé.e dans le service de suites de naissance de la maternité où ils.elles exercent.

A noter que des sorties précoces ont été préconisées par les autorités sanitaires afin de limiter la durée d'hospitalisation des accouchées et des nouveaux-nés pendant la pandémie de Covid 19.

15 Les séances individuelles de préparation à la naissance et à la parentalité ont été annulées ou reportées pour $10.7 \%$ des sages-femmes libéraux.ales et $20.7 \%$ des sages-femmes territoriaux.ales interrogé.e.s lors de l'enquête Covid-SF (Rousseau, A. et al., 2020).

16 Cas de la préparation à la naissance en haptonomie se pratiquant en couple et utilisant la communication par le toucher

17 « Du fait de la crise, des séances de préparation à la naissance ont souvent été annulées. Les sages-femmes hospitalières ont considéré que les patientes étaient moins bien informées/préparées pour les éléments suivants : suivi de la grossesse, gestion de la douleur, motifs de consultation en urgence, accouchement, allaitement, soins de puériculture, contraception » (Rousseau, A. et al., 2020)

18 avec le Collège Français d'Echographie Fœtale qui a co-signé avec le CNGOF un communiqué préconisant l'éviction des accompagnant.e.s pour les échographies prénatales (CFEF, CNGOF, Mars 2020)

19 « Dans chaque région, les maternités élaborent actuellement leur protocole de prise en charge des accouchements. » (CNGOF, 30 mars 2020)

20 « La place de l'accompagnant.e est définie par chaque établissement en fonction de la situation locale. » (CNSF et al., 23 mars 2020)

21 Différents articles de presse font état de l'impact des ressources des maternités sur les décisions d'accueil ou d'éviction des accompagnant.e.s. Exemple : « La polyclinique Majorelle, située à Nancy (Meurthe-et-Moselle), autorise de nouveau la présence du conjoint lors d'un accouchement. Pour assister à la naissance de l'enfant (...) la maternité devra disposer des équipements de protection individuelle (EPI) en nombre suffisant et la personne accompagnante devra porter les mêmes protections que le personnel dans la salle de naissance. » (Ouest France, 2 avril 2020)

22 « La place et le rôle indispensable du conjoint lors de l'accouchement, soulignés et réaffirmés par l'OMS dans le contexte de crise, est aujourd'hui remis en cause du fait du manque de matériel (masques, surblouses...) (...). Les équipes des maternités ont besoin d'équipement suffisant pour que les naissances en France puissent continuer de se dérouler en toute sécurité médicale et émotionnelle pour les équipes soignantes et les couples (...) » Extrait de la lettre ouverte des sages-femmes à Monsieur le Ministre des Solidarités et de la Santé et Monsieur le Directeur Général de la Santé (CNOSF et al., 27 mars 2020)

23 De nombreux articles de presse font état de ces disparités. Exemple : " "Les consignes évoluent, explique Jacky Nizard, président de l'association des gynécologues-obstétriciens européens et obstétricien à la Salpêtrière à Paris. Pour le moment la plupart des maternités acceptent un accompagnant en salle de travail, mais il n'y a pas de règle générale" (...) "Dans environ les trois quarts des maternités d'île de France, les coparents étaient admis en salle de 
naissance. Ensuite, environ deux heures après l'accouchement, au moment de regagner la chambre mère-enfant, ils étaient invités à rentrer chez eux", expliquait le docteur Thierry Harvey, gynécologue obstétricien à la maternité des Diaconnesses, dans le 12e arrondissement de Paris. » (TV5 Monde, 31 mars 2020)

24 « Le papa interdit d'accouchement ? Cette situation, mais aussi la peur d'une contamination en milieu hospitalier, poussent certains couples à envisager l'accouchement à domicile. Les sagesfemmes croulent sous les demandes, toutes ne pourront pas être satisfaites. » (France Soir, 31 mars 2020)

25 " "On a une charge augmentée, avec des demandes vraiment plus importantes ces dernières semaines pour des accouchements à domicile et en maison de naissance" confirme Charlotte Moënnat, sage-femme indépendante à Aubonne. "Les couples se retournent vers nous pour différentes raisons", précise-t-elle : "Je peux relever vraiment une peur liée au virus en milieu hospitalier et l'incertitude parfois de la présence du père à la maternité". » (RTS, 25 mars 2020)

26 « Avec la pandémie, la demande a explosé pour les accouchements à domicile » selon la présidente du Regroupement des sages-femmes du Québec (Beaudoin, D., 17 mai 2020)

27 «"Je ne pense pas qu'il y ait assez de sages-femmes pour faire face à cette hausse des demandes", signale Michelle Palmer, présidente du comité accouchement à domicile de l'association américaine des sages-femmes professionnelles (ACNM). » (AFP, 8 avril 2020)

28 Ailleurs qu'en France, des pétitions sont aussi lancées comme en Suisse, une pétition adressée au CHUV et à la ville de Lausanne "CHUV : Ne pas interdire l'accès aux salles d'accouchement aux futurs papas dû au Covid-19”. (Laufer, S., Mars 2020)

29 Association Nationale des Sages-femmes territoriales, Association Professionnelle des Sagesfemmes, Association Française des Sages-femmes Acupuncteurs, Association Nationale des Sages-femmes Orthogénistes

30 Le CIANE est un collectif d'associations agréé pour représenter les usagers dans le système de santé pour les questions relatives à la grossesse, à la naissance et aux premiers jours de la vie.

31 « Le Syngof vient de publier un communiqué de presse au sujet des recommandations élaborées avec le CNGOF sur le suivi de grossesse et l'accouchement en période de coronavirus. Il est dit dans ce communiqué que « ces recommandations ont reçu l'entière adhésion du Collectif Interassociatif Autour de la Naissance (CIANE)», ce qui n'est pas exact étant donné que le Ciane n'a à aucun moment été consulté ni n'a exprimé un avis sur la question (...). Nous ne nous sommes donc pas prononcées en faveur de tel ou tel protocole, et certainement pas en faveur de l'absence du père. Il nous paraîtrait donc opportun de rappeler que dans la très grande majorité des situations, et non pas seulement parfois, le père peut être présent en salle d'accouchement. » (CIANE, 31 mars 2020)

32 « Au sujet des Fiches Réponses rapides dans le cadre du COVID-19 « Continuité du suivi des femmes enceintes » et « Continuité du suivi postnatal des femmes et de leur enfant » de l'HAS du 2 avril 2020, la Cellule de crise "Covid19-sage-femme » (...) va adresser : • Un courrier à la présidente de la HAS pour demander une révision de ces "réponses rapides" prenant en compte l'avis des professionnels de terrain. - Un courrier au Ministre de la santé et à la DGS pour souligner que ces réponses rapides sont contraires aux recommandations de la DGS, aux communiqués du ministère sur le suivi des femmes enceintes et ignorent les avis des professionnels de terrain. » (Cellule de crise « Covid19-sage-femme », 7 avril 2020)

33 « La place et le rôle indispensable du conjoint lors de l'accouchement, soulignés et réaffirmés par l'OMS dans le contexte de crise, est aujourd'hui remis en cause du fait du manque de matériel (masques, surblouses...) (...). Les équipes des maternités ont besoin d'équipement suffisant pour que les naissances en France puissent continuer de se dérouler en toute sécurité médicale et émotionnelle pour les équipes soignantes et les couples (...) (pour) garantir une prise en charge "décente" et humaine des femmes enceintes, des parturientes, des parents et de leurs enfants ; limitant les séquelles physiques, psychiques et émotionnelles (...).» (CNOSF et al., 27 mars 2020)

34 Après le confinement, à l'occasion du Ségur de la Santé (consultation des acteurs du système de soin français qui se déroule du 25 mai 2020 au 10 juillet 2020), les associations de sagesfemmes exprimeront à nouveau leur sentiment de non prise en considération, disant, en amont, être : «peu connues, souvent oubliées par les institutions. Ce qui vient encore de se confirmer avec le récent « Rapport Claris » (ONSSF, Juin 2020), et, en aval, " avoir été oubliées, alors que la crise, elle, ne nous a pas oubliées » (Raso, A., 31 juillet 2020)

35 Cette perception des sages-femmes et des futurs parents fait écho à l'histoire des épistémologies autour de la naissance. La médicalisation de la grossesse et de l'accouchement a été marquée, dès les XVIIème et XVIIIème siècles, par l'investissement massif de la santé génésique par les chirurgiens devenus ensuite gynécologues obstétricien.ne.s (Gélis, J., 1988 ; Morel, M.F., 1991, 2008) et la « subordination des accoucheuses » puis au XXème siècle par le " grand déménagement » du lieu de la naissance de la sphère domestique vers des espaces médicalisés, publics et institués (Knibiehler, Y., 2016). Si, en France, la santé sexuelle et reproductive des femmes a longtemps été avant tout une affaire de femmes et de sages-femmes, elle est ainsi devenue également une " affaire publique, affaire de médecins " où " l'impératif sécuritaire, joint à la sophistication des savoirs et des savoir-faire, a imposé dans ce domaine la suprématie absolue du corps médical », associée à une perte d'autonomie décisionnelle pour les femmes et les sages-femmes (Knibiehler, Y., 2016). 
36 Référence à Popper : « une décision morale n’a jamais de racine scientifique. En revanche, une décision scientifique a toujours une base morale.

37 Les théories critiques redéfinissent ainsi l'objectivité comme étant articulée avec une subjectivité forte construite collectivement et historiquement. Boaventura de Sousa définira quant à lui l'objectivité comme étant toujours une " intersubjectivité, ou mieux une intersubjectivité auto-consciente »(De Sousa Santos, B., 2018).

38 La version du communiqué actuellement visible sur ce même code URL est une version qui a été modifiée courant juin 2020 (bien que toujours référencée au 30 mars 2020 sur le site). Nous faisons référence dans notre article à la version initiale publiée le 30 mars 2020.

\section{Pour citer cet article}

Référence électronique

Marie-Laure Deneffe Dobrzyński, « Naissance en temps de Covid 19 », Recherches \& éducations [En ligne], HS | Juillet 2020, mis en ligne le, consulté le 09 mars 2021. URL : http://journals.openedition.org/rechercheseducations/11353; DOI :

https://doi.org/10.4000/rechercheseducations.11353

\section{Auteur}

Marie-Laure Deneffe Dobrzyński

Université Paris 8, laboratoire EXPERICE

Doctorante en sciences de l'éducation

Faculté de Médecine et Maïeutique, Université Catholique de Lille

Enseignante Maïeutique

Sage-Femme

\section{Droits d'auteur}

(C) Tous droits réservés 